\title{
A COMPARISON OF TITLE WORDS FOR JOURNAL ARTICLES AND WIKIPEDIA PAGES: COVERAGE AND STYLISTIC DIFFERENCES?
}

\author{
Comparación de palabras de títulos de artículos \\ de revista y páginas de Wikipedia: ¿Diferencias de \\ cobertura y de estilo?
}

\section{Mike Thelwall and Pardeep Sud}

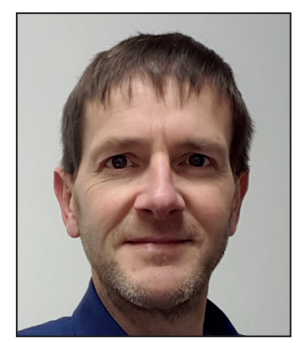

Mike Thelwall leads the Statistical Cybermetrics Research Group at the University of Wolverhampton, UK. He has developed and evaluated free software and methods for systematically gathering and analysing web and social web data, including for sentiment analysis, altmetrics and webometrics, and for Mendeley, Twitter, YouTube, Google Books, blogs and the general Web. He also conducts evaluation exercises for large organisations using web data, including for various divisions within the United Nations and European Commission. He has co-authored hundreds of refereed journal articles and has written three books.

http://www.scit.wlv.ac.uk/ cm1993/mycv.html

https://orcid.org/0000-0001-6065-205X

m.thelwall@wlv.ac.uk

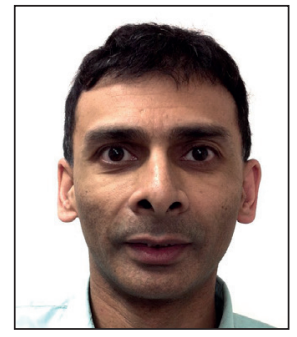

Pardeep Sud is a member of the Statistical Cybermetrics Research Group and a senior lecturer in mathematics and statistics at the University of Wolverhampton, UK. He has published 11 refereed journal articles about alternative indicators for research evaluation. Pardeep also has several years' experience in the actuarial profession, where he specialised in asset-liability modelling for defined benefit pension schemes.

https://orcid.org/0000-0002-3304-0469

p.sud@wlv.ac.uk

University of Wolverhampton, Statistical Cybermetrics Research Group School of Mathematics and Computer Science Wulfruna Street, Wolverhampton WV1 1LY, UK

\begin{abstract}
This article assesses whether there are gaps in Wikipedia's coverage of academic information and whether there are non-obvious stylistic differences from academic journal articles that Wikipedia users and editors should be aware of. For this, it analyses terms in the titles of journal articles that are absent from all English Wikipedia page titles for each of 27 Scopus subject categories. The results show that English Wikipedia has lower coverage of issues of interest to non-English nations and there are gaps probably caused by a lack of willing subject specialist editors in some areas. There were also stylistic disciplinary differences in the results, with some fields using synonyms of "analysing" that were ignored in Wikipedia, and others using the present tense in titles to emphasise research outcomes. Since Wikipedia is broadly effective at covering academic research topics from all disciplines, it might be relied upon by non-specialists. Specialists should therefore check for coverage gaps within their areas for useful topics and librarians should caution users that important topics may be missing.
\end{abstract}

\section{Keywords}

Wikipedia; Research communication; Encyclopedia; Science communication.

\section{Resumen}

Este artículo evalúa si hay vacíos en la cobertura de la información académica de Wikipedia y si existen diferencias estilísticas no obvias entre los artículos de revistas académicas que los usuarios y editores de Wikipedia deben conocer. Para ello se analizan los términos en los títulos de artículos de revistas que están ausentes de todos los títulos de las páginas de Wikipedia en inglés para cada una de las 27 categorías temáticas de Scopus. Los resultados muestran que la Wikipedia en inglés tiene menor cobertura de los temas de interés para las naciones que no son de habla inglesa, y existen lagunas probablemente 
causadas por la falta de editores especialistas dispuestos en algunas áreas. También se encontraron diferencias de estilo según las disciplinas, con algunos campos que utilizan sinónimos de "análisis" que fueron ignorados en Wikipedia, y otros que usan el tiempo presente en títulos para enfatizar los resultados de la investigación. Dado que Wikipedia es muy eficaz en la cobertura de temas de investigación académica de todas las disciplinas, puede ser utilizada por personas no especializadas. Por lo tanto, los especialistas deben verificar las lagunas de cobertura dentro de sus áreas para encontrar temas útiles y los bibliotecarios deben advertir a los usuarios que pueden faltar temas importantes.

Palabras clave
Wikipedia; Comunicación de investigación; Enciclopedia; Comunicación científica.

Thelwall, Mike; Sud, Pardeep (2018). "A comparison of title words for journal articles and Wikipedia pages: Coverage and stylistic differences?". El profesional de la información, v. 27, n. 1, pp. 49-64.

https://doi.org/10.3145/epi.2018.ene.05

\section{Introduction}

Wikipedia, ranked the $5^{\text {th }}$ most popular website in August 2017 by Alexa.com, is a source of a wide variety of mostly accurate information.

http://www.alexa.com/siteinfo/wikipedia.org

Its knowledge is valuable not only for its easy accessibility but also because many people would seek less informative free web alternatives if it did not exist (Fallis, 2008). Wikipedia is widely used in education (e.g., Henderson et al., 2015; Lim, 2009), by the public for health-related issues (e.g., Thomas et al., 2013) and probably also for professional, recreational and other needs. For example, junior doctors may consult Wikipedia regularly (Hughes et al., 2009) and digital archives may link to Wikipedia for contextual information (Szajewski, 2013). Although institutions and research funders finance open access journal articles to make academic knowledge available to all (Lange, 2016; Pinfield; Salter; Bath, 2016), scholarly topics on Wikipedia may well be consulted by a wider section of the population than read journal articles. It is therefore important to understand how Wikipedia covers academic information and assess the comprehensiveness of its coverage (e.g., Rush; Tracy, 2010). For example, Cochrane is working with WikiProject Medicine to ensure that, when possible, Wikipedia articles on medical topics are supported by state of the art evidence from Cochrane reviews (Mathew et al., 2013).

https://en.wikipedia.org/wiki/Wikipedia:WikiProject_Medicine

\section{Although Wikipedia covers many acade-} mic research topics, it is not known whether it has substantial gaps in content

Although Wikipedia covers many academic research topics (Mesgari et al., 2015), it is not known whether it has substantial gaps in content. If such gaps were found, then researchers and research funders may consider taking extra steps to ensure that steps are taken to remedy this issue.

Wikipedia articles on specialist research topics are presumably often written or edited by field specialists or postgraduate students that are casual editors of Wikipedia rather than experienced Wikipedians. It would also therefore be useful to gain insights into any aspect of the way in which Wikipedia's style differs from that of journal articles. This could help casual editors to tailor their style when contributing to Wikipedia or when translating academic research.

The two goals of this article are to get insights into (but not comprehensively evaluate):

a) the extent to which Wikipedia covers academic research and

b) stylistic differences in presentation between Wikipedia and journal articles.

Both are addressed by comparing the words used in the titles of academic articles with the words used in Wikipedia page titles. Of course, there are likely to be major obvious differences between the two due to their different functions. Nevertheless, this approach can give insights into gaps in Wikipedia and stylistic difference between the two sources of information across many different fields. It was chosen as a practical way to make large scale comparisons between Wikipedia and academia, although many areas of scholarship are not covered. Article keywords could also have been analysed but these are less rich than title words and for some journals are restricted to controlled vocabularies, such as MeSH, that may be out of date or change the focus of the study to the controlled vocabulary itself. Since article keyword styles differ between journals (different controlled vocabularies, different controlled vocabulary versions, nonuse of controlled vocabularies), any interdisciplinary analysis of these would be necessarily complex and would not be able to give fully comparable results between disciplines.

\section{Wikipedia}

An encyclopedia is,

"A literary work containing extensive information on all branches of knowledge, usually arranged in alphabetical order",

or

"An elaborate and exhaustive repertory of information on all the branches of some particular art or department of knowledge; esp. one arranged in alphabetical order" (OED, 2016).

Wikipedia is an example of the former kind because its co- 
verage is not restricted by topic. Whilst earlier forms were conceived as comprehensive knowledge for education (Lærke, 2014), later encyclopaedias serve more of a reference function - hence perhaps the shift from subject-based groupings of topics to an alphabetical list of entries (Loveland, 2013).

https://www.britannica.com/topic/encyclopaedia

Wikipedia's model of user-edited content seems to have largely eclipsed previous encyclopedias due to its more comprehensive free coverage (Gralla, 2009) combined with a similar level of accuracy (Giles, 2005; Mesgari et al., 2015; Stankus; Spiegel, 2010), albeit with less credibility (Flanagin; Metzger, 2011; Kubiszewski; Noordewier; Costanza, 2011; but see: Gorichanaz, 2016). Its coverage may be less accurate for topics that attract non-expert participation, such as those that are politically sensitive (Wilson; Likens, 2015). There is some evidence that editors can be casual about citing sources (Luyt, 2015). Wikipedia does not cover news, however.

https://en.wikipedia.org/wiki/Wikipedia:What_Wikipedia_is_not

A consequence of the differences between Wikipedia and a traditional encyclopaedia is that its contents and coverage style are likely to differ. For example, in comparison to the UK-based Encyclopedia Britannica, Wikipedia articles about large businesses seem to express more sentiment, be longer and to cover ethical issues more (Messner; DiStaso, 2013).

Wikipedia's unpaid contributors tend to act for their own personal reasons rather than from an external imperative (Yang; Lai, 2010) and enjoy contributing (Nov, 2007). They are rarely motivated by a need for public recognition, but tend to believe that the activity is useful, that they are competent to edit, and that contributing is fair because they also use the information (Cho; Chen; Chung, 2010; Lai; Yang, 2014). Another motivation is to support personal development (Xu; Li, 2015). There do not seem to be any studies of the reasons why researchers contribute to Wikipedia, but the need to organise to ensure high quality coverage of academic-related topics is evident in initiatives like WikiProject Medicine.

\section{Wikipedia coverage may be less accurate} for topics that attract non-expert participation, such as those that are politically sensitive

\subsection{Wikipedia content}

Wikipedia's size allows it to subsume the functions of a specialist encyclopedia. These have historically allowed a more detailed treatment of a single broad subject area of cultural (e.g., from the 1732 Musikalisches Lexikon to the modern "companions" to areas of literature from Oxford and Cambridge) or scientific interest (e.g., from the 1821 Dictionary of Chemistry to modern handbooks for areas of research produced by scholarly publishers). For instance, an encyclopedia for a field may be expected to summarise its important features (e.g., Dick, 2015), sometimes keeping this up to date by using digital formats (Remy, 2015). The restric- tion to a specialist area probably allows a more technical language to be used.

One issue that Wikipedia may not be good at dealing with is the need to provide comprehensive information about topics from the perspective of all relevant types of user

One issue that Wikipedia may not be good at dealing with is the need to provide comprehensive information about topics from the perspective of all relevant types of user. For example, if Wikipedia is relied upon in education then gaps in coverage could cause problems to students that rely upon it (Azer, 2015). Wikipedia pages are also widely cited by patents, often using them to support knowledge claims (Orduña-Malea; Thelwall; Kousha, 2017) and so gaps in Wikipedia may translate to problems in patents. Whilst a casual editor may be likely to make accurate edits, they may not be well positioned to judge whether important information is missing. Medical information is particularly critical in this regard on Wikipedia because article inaccuracies or omissions may have serious health consequences (Hasty et al., 2014; Masukume et al., 2016). There are also cultural biases in the content of articles. Biographies of individuals vary between different language versions of Wikipedia (Callahan; Herring, 2011) and businesses are more extensively discussed in Wikipedia articles within their own languages (Roessing; Einwiller, 2016). The term "bias" here is used in a descriptive rather than pejorative sense.

Many previous studies have analysed the comprehensiveness or accuracy of Wikipedia for specific topics (Mesgari et al., 2015) but there have been no recent empirical assessments of its relationship with academic knowledge with wide coverage. It does not have systematic and appropriate coverage of scholars (Samoilenko; Yasseri, 2014) and some broad subject areas were under-represented compared to books in 2006, with gaps in content compared to subject-specific encyclopedias (Halavais; Lackaff, 2008). Popular and more current topics also have longer articles (Royal; Kapila, 2009).

\subsection{Wikipedia and academic journal articles}

The most obvious difference between Wikipedia and an academic journal article is that Wikipedia does not allow pages to report original research.

https://en.wikipedia.org/wiki/Wikipedia:No_original_research

Wikipedia also attempts to summarise knowledge, and by extension to exclude unnecessary fine details, whereas only academic journal articles that are literature reviews have knowledge summarisation as a primary function.

https://en.wikipedia.org/wiki/Wikipedia:Scope https://en.wikipedia.org/wiki/Wikipedia:Too_much_detail

An academic journal article may be expected to contain details that will not inform the general reader but would be important for other researchers, such as information that would allow an experiment to be reproduced. Wikipedia articles should be targeted at general readers and should 
therefore ignore specialist terminology and assumed prior knowledge as far as possible, differing in this respect from journal articles.

https://en.wikipedia.org/wiki/Wikipedia:What_Wikipedia_is_not

Whilst a casual editor may be likely to make accurate edits, they may not be well positioned to judge whether important information is missing. Medical information is particularly critical in this regard

The Encyclopedia Britannica claims that an encyclopedia typically summarises published scholarship, which suggests a close relationship with academic research and would exclude most popular culture information.

https://www.britannica.com/topic/encyclopaedia

In contrast, Wikipedia grew based on anyone being able to add content, without the need to be an expert on the subject area and without the need for this content to be reviewed by a trusted person (De-Laat, 2012). This opens the door to non-academic content and popular culture (Yam, 2016). Nevertheless, at least one journal, PLoS Computational Biology, has explicit Wikipedia-friendly policies to help make high quality research-informed information more accessible. This includes publishing Wikipedia-friendly versions of articles (Wodak et al., 2012). Other academics have made specific pleas for disseminating an area of scholarship via Wikipedia (Signore; Serio; Santamaria, 2014).

The content of Wikipedia has previously been compared to published academic research. Journals cited by English language Wikipedia articles in April 2007 were compared to those listed in the Journal Citation Reports (JCR) multidisciplinary set (Nielsen, 2007; see also: Kousha; Thelwall, 2017). From the 30,368 matches found, higher impact journals were more likely to be cited (e.g., Nature: 787; Science: 669$)$ and medical and astronomy journals seemed to be overrepresented. There was also at least one active area within Wikipedia with many citations to relatively obscure journals, such as Australian Banksia species articles citing Australian botany journals, but this study did not report a systematic comparison of academic research topics and Wikipedia content. A study of 4721 journals in 26 fields confirmed that articles were more likely to be referenced if they were in higher impact journals but also found that open access journals were more likely to be cited (Teplitskiy; Lu; Duede, 2017).

A topic-based investigation of citations in Wikipedia articles focused on one of its high-profile areas, astronomy. Older research was less cited in Wikipedia than newer research (Thelwall, 2016), suggesting that shifts in the focus of academic research could lead to changes in the attention given to different topics. High profile issues, such as the planet status of Pluto, seemed to result in an increase in Wikipedia editor activity. A study of wind power pages in Wikipedia found that a quarter of their references cited academic publications but, less than $1 \%$ of academic wind power articles had been cited in wind power Wikipedia pages (Serrano-López; Ingwersen; Sanz-Casado, 2017). If typical, this suggests that the vast majority of individual academic papers are ignored by Wikipedia.

\subsection{Wikipedia and academic article title styles}

Wikipedia page titles can be either a name or a topic description, according to the official guidelines. They should be recognisable, natural, precise, and concise, as well as being consistent with the titles of similar articles. Disambiguation pages can be used for cases where different topics could have the same name. There are also some specific conventions for naming types of articles, such as books, people, organisations and events.

https://en.wikipedia.org/wiki/Wikipedia:Article_titles

Academic journal article titles have more flexibility than Wikipedia pages, although some journals and field norms may be prescriptive. They should ideally summarise the content of an article to help someone decide whether to read the abstract for more information (Swales, 1990). The most common formats are declarations of results or descriptions of the paper (Jamali; Nikzad, 2011). Titles may express the purpose or results of a study, its methods (Méndez; Alcaraz; Salager-Meyer, 2014; Paiva; Lima; Paiva, 2012) or the overall study design (Ubriani; Smith; Katz, 2007). A descriptive chemistry title, for example, may centre around variants of the phrase "an analysis of" (Sano; Fujiwara, 1993). Nevertheless, authors may be inefficient or ignore the function of article titles (Hartley, 2005) and can also find creative solutions by adopting or inventing alternative strategies to attract attention (Hartley, 2007). Titles can be questions, but these are rare in all disciplines (Cook; Plourde, 2016; Méndez; Alcaraz; Salager-Meyer, 2014) although they are increasing in frequency (Ball, 2009).

\section{Wikipedia grew based on anyone being able to add content, without the need to be an expert on the subject area and without the need for this content to be reviewed by a trusted person (De-Laat, 2012)}

Acronyms can also be present in article titles (Rostami; Mohammadpoorasl; Hajizadeh, 2014) but these can also occur in Wikipedia redirection and disambiguation page titles even if they are rare in standard Wikipedia page titles. Present participles (verbs ending in -ing) can also be used to emphasise the importance of results (Wang; Bai, 2007) and these seem likely to be absent from Wikipedia titles since they do not need to perform this function. The same is true for past participles (Wang; Bai, 2007).

There are disciplinary differences in the constructions of article titles. For example, complete sentences are more common in some disciplines than others and the use of compound titles, such as with a colon in the middle, is particularly common in the social sciences (Soler, 2011). Compound titles may start with a general theme and then finish 
with a particularising aspect (Hartley, 2007) and terms playing the latter role may be too specific for a Wikipedia page. Older fields may also tend to have longer article titles (e.g., White; Hernandez, 1991) as articles become more specialised to fill gaps left by previous research.

\section{Research hypotheses}

The following hypotheses drive the research. They are partly informed from the literature review above and partly by initial explorations of the data. The primary goal of the paper is to seek evidence of coverage gaps, which relate primarily to hypotheses 4 and 5 , and the secondary goal is to seek evidence of stylistic differences, which relate to hypotheses 1,2 , and 3 . The secondary goal also aids the primary goal by separating term differences that are not due to coverage gaps.

\section{Hypothesis 1 (complexity)}

Terms that are common in Scopus article titles for a subject but absent from Wikipedia titles are often complex in format or in the ideas represented. This seems likely because encyclopedias summarise knowledge for the non-expert (Béjoint, 2000) and therefore seem likely to use simpler language than that of journal articles, which target subject experts or specialists. Moreover, one form of complexity is specificity and so articles may be about topics that are too specific to merit their own Wikipedia page.

\section{Hypothesis 2 (research process descriptions)}

Terms that are common in Scopus article titles for a subject but absent from Wikipedia titles include terminology that describes the research process (e.g., Méndez; Alcaraz; Salager-Meyer, 2014; Paiva; Lima; Paiva, 2012; Ubriani; Smith; Katz, 2007). This seems likely because the research process is important in academia but research outcomes are more relevant to the summarising functions of an encyclopedia. Nevertheless, the research process itself can be an object of study and hence may be described in separate Wikipedia pages.

\section{Hypothesis 3 (stylistic and structural differences)}

Some terms that are common in Scopus article titles for a subject but absent from Wikipedia titles are the result of stylistic differences in the way in which academic titles are formed (e.g., Jamali; Nikzad, 2011) in comparison to Wikipedia page titles, or structural differences in the types of entities that are given their own pages.

\section{Hypothesis 4 (culture bias)}

Terms that are common in Scopus article titles for a subject but absent from Wikipedia titles include terminology that is geographically or culturally tied to languages other than English (see: Roessing; Einwiller, 2016). This will be most evident in the arts and humanities (even though scholars tend to write in their own language, humanities deal with many location-specific phenomena). It will also occur in sciences that deal with location-specific phenomena, such as geography, social sciences and applied sciences. It will not be evident in pure sciences.

\section{Hypothesis 5 (editor gaps)}

There are gaps in the coverage of Wikipedia that are reflected in some important subject terms being absent from Wikipedia page titles. These seem likely to occur because the voluntary nature of Wikipedia and the need for specialists to edit academic topics means that areas lacking specialist volunteers may lack content or may omit content that is relevant to some users (Azer, 2015).

\section{Methods}

The research hypotheses were addressed by identifying and manually examining a large set of words that frequently occur in academic journal article titles but rarely in Wikipedia page titles. To start, different Scopus subject categories were chosen to represent a wide range of academic fields. Scopus categories were chosen as a transparent method of collecting together large and reasonably coherent collections of subject-based journal articles. Although some journals are interdisciplinary or occasionally publish articles from out of their core scope, this is unlikely to affect the primary research method, as described below. Scopus was selected in preference to the Web of Science for its finer grained categories. The seventh field was selected from each broad Scopus category, replacing this with the next alternative (counting in cycles) for categories with less than 7 subjects. This produced a set of 27 subject categories from diverse fields from the arts and humanities, social sciences, engineering, formal sciences, physical sciences, life sciences, medicine and health science (Table 1).

The titles of all journal articles published between 1996 and 2015 (20 years) were downloaded from Scopus. The year 1996 was the starting date because the coverage of Scopus expanded in this year. English language articles dominate Scopus and non-English articles were not excluded because some topics of international interest may be primarily discussed in other languages (e.g., Spanish, German, French, Chinese). This might have resulted in non-English function words occurring in the stylistic analyses but this did not occur due to the dominance of English in Scopus titles and abstracts. The final year was 2015 to ensure that there had been sufficient time (almost 1 year) for all articles to have been added to Wikipedia. There was a system limit of 10,000 articles per year and in large subject areas and more recent years, this resulted in incomplete coverage. In such cases, only the first and last 5000 articles from the subject and year were obtained. This should not have a major impact on the results or bias them, given the large total numbers involved. For each of the 27 subjects, a complete list was built of all words in all titles, together with their frequency. The free software Webometric Analyst was used for this [Tab-sep menu; Count frequency of words in text or column $n$ (e.g., Wikipedia titles) menu item].

The titles of all Wikipedia articles were extracted from a data dump of Wikipedia from December 12, 2016 of a "List of all page titles in the main namespace". https://dumps.wikimedia.org/enwiki

This includes standard page titles and disambiguation pages, which are the main pages that typical visitors will see. 
It excludes meta/background pages, such as those for editor discussions and image files.

https://meta.wikimedia.org/wiki/Help:Namespace

All words were extracted from all these Wikipedia page titles and used to form a word frequency list in the same way as for the Scopus article titles. This resulted in 2,417,043 different words with frequencies up to $1,064,797$ (of).

No word stemming was applied to either term list because the focus of the study is on academic language, which by its nature involves rare words for which the normal rules of grammar may not apply. Moreover, given that the comparisons span very large word lists, even rare systematic errors can impact on the word frequency comparison results. Hyphens in the middle of words or at their end were retained within the words. Hyphens are an important part of names in some fields (Burke, 2008; Frey-Klett et al., 2011; Gill et al., 2009).
For each of the 27 subjects, each term in the term frequency list was checked against the Wikipedia list and the presence or absence of a match was recorded. The 25 most frequent words in the subject that were absent from the Wikipedia data were then saved for manual analysis, resulting in 27 sets of 25 terms (675 in total). These are therefore all words that are frequent in article titles for a specific Scopus subject category but completely absent from Wikipedia page titles. For example, "mucoadhesive" occurred 474 times in Scopus journal articles from the category Pharmaceutical Science but not in any Wikipedia page titles.

The primary analysis was an investigation into the linguistic properties of the 675 terms extracted. Although the goal of this paper is to assess academic knowledge gaps, initial testing indicated strong linguistic commonalities that were relevant to the research hypotheses. For the main manual analysis, the 27 sets of 25 terms were manually examined and their

Table 1. The 27 Scopus subject categories examined and the number of articles extracted from them for the years 1996-2015 (as of December 2016). The Table includes equivalent information for Wikipedia (bottom row). The top term is the most common term in article titles that is not in a Wikipedia page title. See Table 2 for the codes used.

\begin{tabular}{|c|c|c|c|c|c|}
\hline Subject area & Articles & Title words & Mean title words & SD title words & Top term \\
\hline App Microbiol \& Biotech. & 171,555 & $2,266,862$ & 13.2 & 4.63 & Enhances (PT) \\
\hline Atomic \& Mol Phys \& Optics & 199,990 & $2,089,197$ & 10.4 & 4.18 & All-optical (Hy) \\
\hline Cell Biology & 199,308 & $2,600,433$ & 13.0 & 4.76 & Regulates (PT) \\
\hline Comp. Vis. \& Pattern Recog. & 70,807 & 641,427 & 9.1 & 3.58 & Wavelet-based (Hy) \\
\hline Control \& Systems Eng. & 177,184 & $1,661,292$ & 9.4 & 3.52 & Observer-based (Hy) \\
\hline Dental Assisting & 89 & 700 & 7.9 & 3.45 & Perimplantitis (Su) \\
\hline Dermatology & 140,309 & $1,500,975$ & 10.7 & 5.39 & HIV-infected (Hy) \\
\hline Discrete Math \& Combin. & 51,666 & 394,323 & 7.6 & 3.51 & Subgraphs (PI) \\
\hline Emergency Nursing & 16,082 & 147,366 & 9.2 & 5.46 & Out-of-hospital (Hy) \\
\hline Endocrine \& Autonomic & 14,195 & 188,404 & 13.3 & 4.69 & Modulates (PT) \\
\hline Finance & 90,791 & 820,166 & 9.0 & 3.95 & Spillovers (Co) \\
\hline Fluid Flow \& Transfer Proc & 85,996 & 961,890 & 11.2 & 4.41 & Impinging (PP) \\
\hline Forestry & 96,934 & $1,257,994$ & 13.0 & 4.51 & Provenances (PI) \\
\hline Fuel Technology & 148,800 & $1,646,493$ & 11.1 & 4.31 & Non-premixed (Hy) \\
\hline Geology & 151,359 & $1,979,169$ & 13.1 & 4.94 & Ore-forming (Hy) \\
\hline Health, Toxicology \& Mut & 117,852 & $1,583,038$ & 13.4 & 4.75 & Subchronic (Pr) \\
\hline History \& Philosophy of Sci & 29,996 & 291,535 & 9.7 & 4.96 & Preservice (Pr) \\
\hline Human Factors \& Ergonom & 21,378 & 233,041 & 10.9 & 4.36 & Quantifying (PP) \\
\hline Medical Laboratory Tech & 17,600 & 213,768 & 12.1 & 5.40 & Clinicopathologic (Co) \\
\hline Org Behav \& Hum Res Man & 56,732 & 567,651 & 10.0 & 4.24 & Moderating (PP) \\
\hline Pharmaceutical Science & 171,798 & $1,972,024$ & 11.5 & 4.82 & Dispersions (PI) \\
\hline Polymers \& Plastics & 199,852 & $2,165,758$ & 10.8 & 4.21 & Hyperbranched (Pr) \\
\hline Small Animals & 9,204 & 105,265 & 11.4 & 5.51 & Frozen-thawed (Hy) \\
\hline Social Psychology & 94,661 & $1,055,939$ & 11.2 & 4.47 & Moderating (PP) \\
\hline Spectroscopy & 172,971 & $2,205,312$ & 12.8 & 4.93 & Preconcentration (Pr) \\
\hline Stats, Prob \& Uncertainty & 65,796 & 576,474 & 8.8 & 3.60 & Change-point (Hy) \\
\hline Transplantation & 77,971 & $1,006,897$ & 12.9 & 4.89 & Undergoing (PP) \\
\hline Scopus 27 & $2,650,876$ & $30,133,393$ & 11.0 & 4.50 & \\
\hline Wikipedia & $12,922,668$ & $35,370,976$ & 2.7 & 1.86 & \\
\hline
\end{tabular}


key linguistic features were extracted and compared. This was achieved by the first author reading the lists, identifying factors in common that were relevant to the research hypotheses and then classifying all the terms for these factors. These categories are described in the results section. This process was repeated until no further common factors could be identified. A native English-speaking coder independent of the project and with a degree in English independently re-classified 100 of the texts using the guidelines in the table caption and examples from the first coder, achieving a Cohen's kappa value of 0.836 for inter-coder consistency (Cohen, 1960) for 87\% agreement. Cohen's kappa assesses the agreement between two coders on the same categorisation task, factoring out chance rates of agreement. Values above 0.8 could be described as "almost perfect agreement" (e.g., Landis; Koch, 1977). Even accounting for chance rates of agreement, this indicates that the two coders almost always agreed. This confirms that the classification scheme is transparent and straightforward, with only a small element of subjectivity.

For a secondary cross-check, when possible, key properties identified from the main manual analysis were followed up by comparing relevant properties for the set of terms from the subject that were not found in Wikipedia with the corresponding set of terms from the subject that were also in Wikipedia. This comparison allows a check of whether any differences identified also occur outside of the top 25 terms, and that they are genuinely the result of differences with Wikipedia.

\section{Results}

A total of 2,650,876 articles were extracted from the 27 subject categories, but the Dental Assisting category is too smaII to give useful data (Table 1). The full data for this article is available online:

https://figshare.com/s/1e8774053297ddd4257e

Journal article titles were typically longer (11.0 words) than Wikipedia page titles (2.7 words). The length difference is probably due to the many Wikipedia pages that are nouns or noun phrases, such as names of people (e.g., Tom Tureen), categories toward the right.
Table 2. Characteristics of the 25 terms that are most frequent in article titles but absent from Wikipedia titles. $\mathrm{Cu}=$ related to non-English-speaking cultures; PT=present tense verbs; PP=present participle verbs (-ing, but gerund or a verbal noun); $\mathrm{Hy}=$ hyphenated terms; $\mathrm{Co}=$ nominal compound or acronym, $\mathrm{Po}=$ possessives;

\begin{tabular}{|c|c|c|c|c|c|c|c|c|c|c|}
\hline Subject area & $\mathrm{Cu}$ & PT & PP & Hy & Co & Po & $\mathbf{P I}$ & Pr & Su & Ot \\
\hline App. Microb. \& Biotech. & 0 & 8 & 0 & 5 & 4 & 0 & 3 & 2 & 3 & 0 \\
\hline At. \& Mol. Physics, Optics & 0 & 0 & 0 & 14 & 3 & 0 & 3 & 2 & 3 & 0 \\
\hline Cell Biology & 0 & 18 & 0 & 4 & 3 & 0 & 0 & 0 & 0 & 0 \\
\hline Comp. Vis. \& Pattern Rec. & 0 & 0 & 2 & 17 & 2 & 0 & 0 & 2 & 2 & 0 \\
\hline Control \& Systems Eng. & 0 & 0 & 0 & 20 & 1 & 0 & 2 & 1 & 1 & 0 \\
\hline Dental Assisting & 3 & 0 & 1 & 7 & 2 & 3 & 2 & 3 & 3 & 1 \\
\hline Dermatology & 0 & 7 & 3 & 7 & 4 & 0 & 0 & 3 & 1 & 0 \\
\hline Discr. Math. \& Comb. & 0 & 0 & 2 & 10 & 0 & 0 & 11 & 1 & 0 & 1 \\
\hline Emergency Nursing & 1 & 1 & 1 & 12 & 2 & 0 & 1 & 3 & 3 & 1 \\
\hline Endocrine \& Auto. Sys. & 0 & 14 & 1 & 8 & 1 & 0 & 0 & 0 & 1 & 0 \\
\hline Finance & 1 & 1 & 2 & 9 & 0 & 3 & 6 & 2 & 1 & 0 \\
\hline Fluid Flow \& Trans. Proc. & 0 & 0 & 0 & 15 & 3 & 0 & 2 & 3 & 2 & 0 \\
\hline Forestry & 0 & 0 & 2 & 13 & 4 & 0 & 3 & 2 & 1 & 0 \\
\hline Fuel Technology & 0 & 0 & 0 & 12 & 6 & 0 & 2 & 4 & 1 & 0 \\
\hline Geology & 3 & 0 & 1 & 9 & 4 & 0 & 3 & 3 & 2 & 0 \\
\hline Health, Tox. \& Mut. & 0 & 6 & 1 & 9 & 4 & 0 & 2 & 3 & 0 & 0 \\
\hline History \& Phil. of Sci. & 0 & 0 & 7 & 4 & 1 & 3 & 2 & 4 & 3 & 1 \\
\hline Human Factors \& Erg. & 0 & 0 & 3 & 9 & 6 & 0 & 2 & 2 & 3 & 0 \\
\hline Medical Lab. Tech. & 0 & 1 & 2 & 6 & 8 & 0 & 0 & 8 & 0 & 0 \\
\hline Org. Behav, \& HRM & 1 & 1 & 2 & 14 & 1 & 6 & 0 & 0 & 0 & 0 \\
\hline Pharmaceutical Science & 0 & 5 & 0 & 7 & 5 & 0 & 4 & 0 & 3 & 1 \\
\hline Polymers \& Plastics & 0 & 0 & 0 & 8 & 2 & 0 & 4 & 7 & 4 & 0 \\
\hline Small Animals & 0 & 1 & 1 & 9 & 5 & 0 & 1 & 4 & 4 & 0 \\
\hline Social Psychology & 0 & 1 & 2 & 9 & 1 & 6 & 1 & 1 & 4 & 0 \\
\hline Spectroscopy & 0 & 0 & 0 & 10 & 9 & 0 & 2 & 3 & 1 & 0 \\
\hline Stats, Prob. \& Uncert. & 0 & 0 & 3 & 14 & 1 & 0 & 3 & 2 & 1 & 1 \\
\hline Transplantation & 0 & 6 & 1 & 9 & 4 & 0 & 0 & 5 & 0 & 0 \\
\hline Overall average & 0.3 & 2.6 & 1.4 & 10.0 & 3.2 & 0.8 & 2.2 & 2.6 & 1.7 & 0.2 \\
\hline Overall percentage & $1 \%$ & $10 \%$ & $5 \%$ & $40 \%$ & $13 \%$ & $3 \%$ & $9 \%$ & $10 \%$ & $7 \%$ & $1 \%$ \\
\hline
\end{tabular}

animals or plants (e.g., Yucatan Jay), objects (e.g., SS King James), places (e.g., Senaki District), concepts (e.g., Religious Satanism) and cultural products (e.g., Dangerous Girls).

Each of the top 25 terms in a Scopus journal article title but absent from all Wikipedia page titles were placed in one of ten categories according to the likely reason for their absence: $\mathrm{Cu}=\mathrm{of}$ primary interest in non-English-speaking cultures; PT=present tense verbs; $\mathrm{PP}=$ present participle verbs (-ing, but not gerund or a verbal noun); $\mathrm{Hy}=$ hyphenated terms; $\mathrm{Co}=$ nominal compound term (i.e., with two separate major word parts, such as vibrotactile but not microring because micro- is a suffix) or acronym; Po=possessives; Pl=plurals; $\mathrm{Pr}=$ term with prefix (e.g., micro-, di-, nano-); Su=term with suffix (e.g., -ive, -ed, -ological); Ot=others. Categories toward the left have priority over categories toward the right, with each word being allocated to the leftmost category that it fits. This order was chosen to reveal the most important patterns in the data. 
The key attributes of the categories are described below, matched with the hypotheses that they are most relevant for, although some relate to multiple hypotheses.

\subsection{Hypothesis 1: Complexity}

Compound and hyphenated terms are indicators of complexity at the word level because they bind together different concepts. The same is true for possessives since they connect to another concept in the title.

\section{Compound and hyphenated} terms form a majority, on average, of the 25 terms for each subject that are most frequent in article titles but absent from Wikipedia page titles (Figure 1; Table 2). These take the form of hyphenated words (40\% overaII) or non-hyphenated compound words (13\% overall) both of which are complex in the sense of merging together two distinct substantial terms. This type of term was particularly common in Control and Systems Engineering (84\%), including five '-based' terms and two '-feedback' and '-dependent' terms. In this area, hyphenated terms seem to be primarily useful as adjectives to describe the distinctive features of a control system. Compound and hyphenated terms were least common in History and Philosophy of Science (20\%).

Comp. Vis. \& Pattern Rec. At. \& Mol. Physics, Optics Discr. Math \& Comb.

Control \& Systems Eng. Stats, Prob. \& Uncert. Fuel Technology

Human Factors \& Erg.

Endocrine \& Auto. Sys. Polymers \& Plastics

Fluid Flow \& Trans. Proc. $\begin{array}{rr} & \text { Spectroscopy } \\ \frac{0}{0} & \text { Cell Biology }\end{array}$

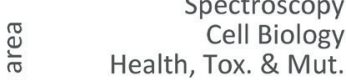
Transplantation Finance

Emergency Nursing Forestry Org. Behav, \& HRM App. Microb. \& Biotech. Geology Dermatology Social Psychology Medical Lab. Tech. Pharmaceutical Science Small Animals

History \& Phil. of Sci. Dental Assisting

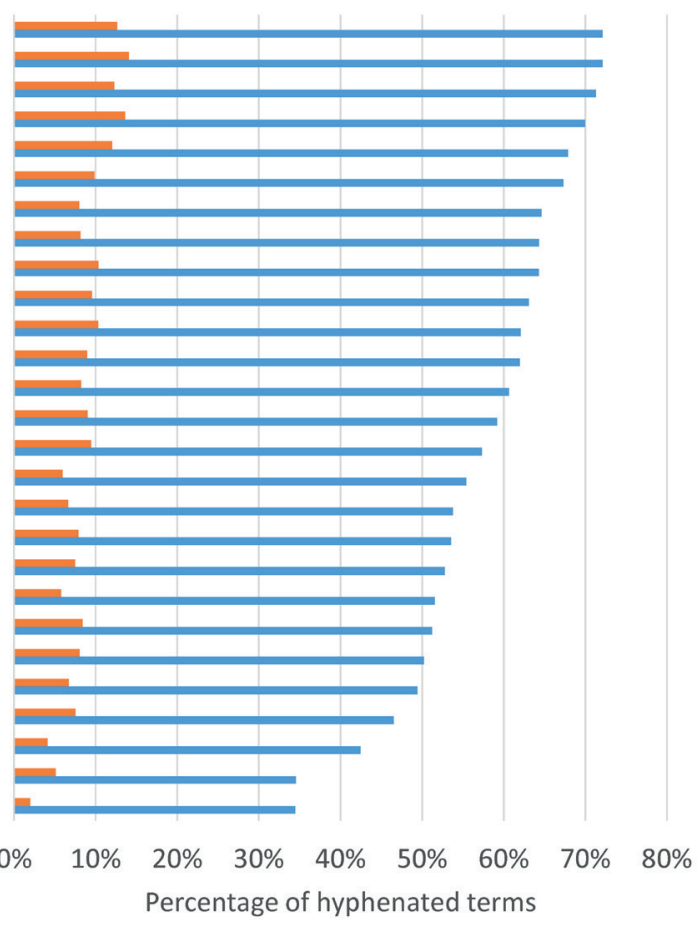

In Wikipedia Not in Wikipedia

Figure 2. The percentage of terms in the titles of Scopus articles that are hyphenated, broken down by whether they are also in Wikipedia.
In three subjects, several of the most frequent hyphenated terms had a common term. Seven Computer Vision and Pattern Recognition terms had the same second part (wavelet-based, feature-based, gradient-based, HMM-based, appearance-based, block-based, SVM-based) for descriptions of the key features of algorithms. In Endocrine and Autonomic Systems three terms had the same second part (anxiety-like, depressive-like, depression-like), for describing symptoms. In Social Psychology there were three terms with a common first part (self-perceptions, self-other, self-reports) and two other terms included a specific role (parent-adolescent, mother-child). This aligns with the social psychology focus on individuals in a social context, but it is not clear why these terms would not be in a Wikipedia page title.

Possessives were rare overall (3\%: Table 2) but were the most common in the two disciplines with a focus on human behaviour in groups. Perhaps unsurprisingly, the Organizational Behavior and Human Resource Management possessives related to organisational groups (firms', auditors', followers', subordinates', organizations', managers') whereas the Social Psychology possessives were more general or more to non-work contexts (couples', preschoolers', therapists', adolescents', individuals', jurors') but again this does not explain their absence from Wikipedia. Possessives function to bind words together and so are an additional complexity flag. Possessives played a different role in 
two other subjects. For History and Philosophy of Science they were exclusively used to refer to academic theories (Carnap's, Vygotsky's, Bourdieu's). For the tiny Dental Assisting subject, the terms were mixed (therapy's, hygienist's, hands').

Overall, there is strong evidence of complexity in the top 25 terms, with a majority being explicitly the combination of two or more words. For the largest category, hyphenated terms, those that are also in Wikipedia are rare compared to those that are not for the full data sets (Figure 2). They also form a majority of terms absent from Wikipedia page titles in most subject categories. Thus, hyphenation is a universal major difference between journal article titles and Wikipedia page titles.

Possessives are relatively rare and in the full data sets are usually, but not always, not found in Wikipedia page titles (Figure 3). There are huge disciplinary differences in the prevalence of possessives. In the subject areas for which they are most common, possessives are mostly absent from Wikipedia page titles (as far down as Transplantation in Figure 3). There is a general tendency for people focused disciplines to include more possessives,

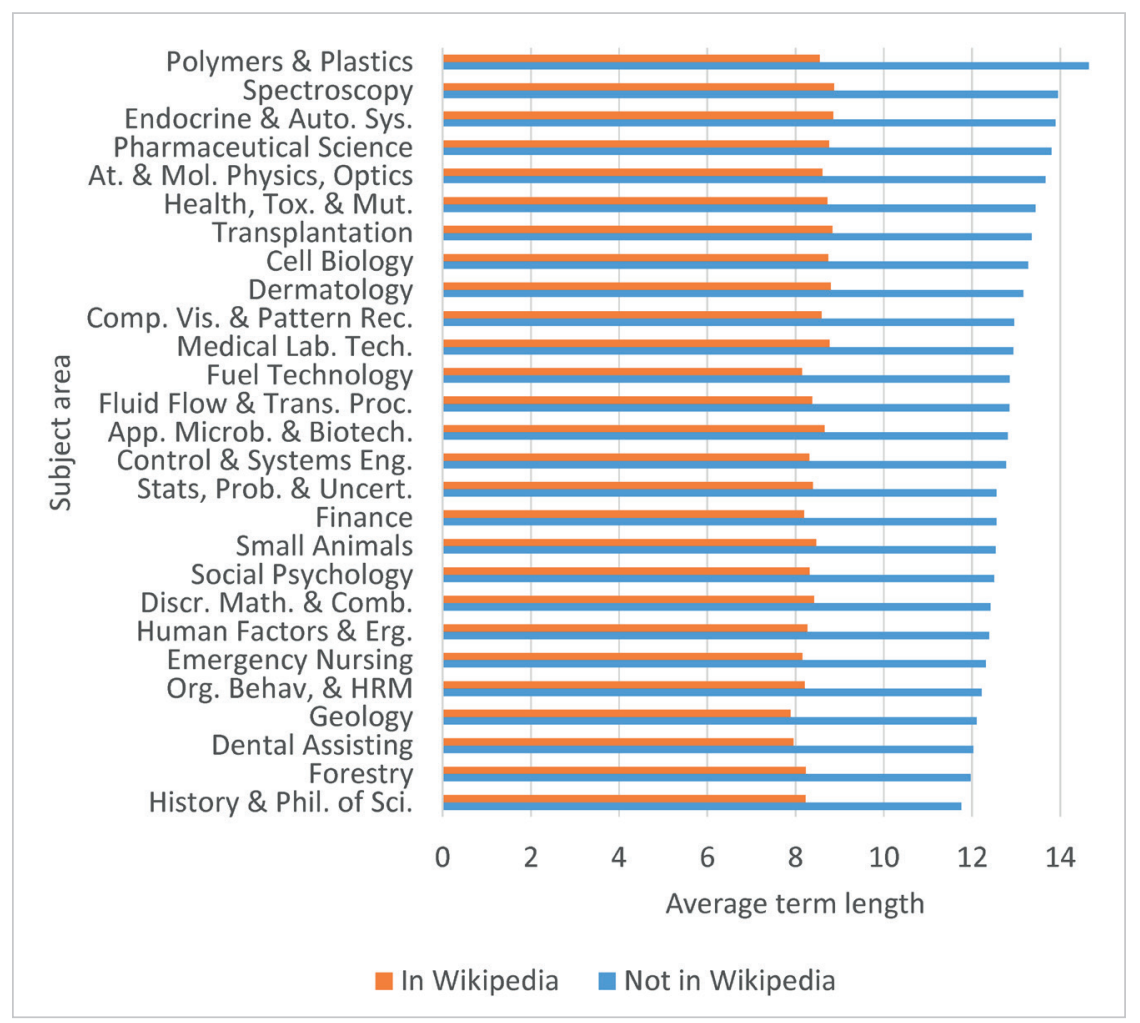

Figure 4. The average length of terms in the titles of Scopus articles broken down by whether they are also in Wikipedia.

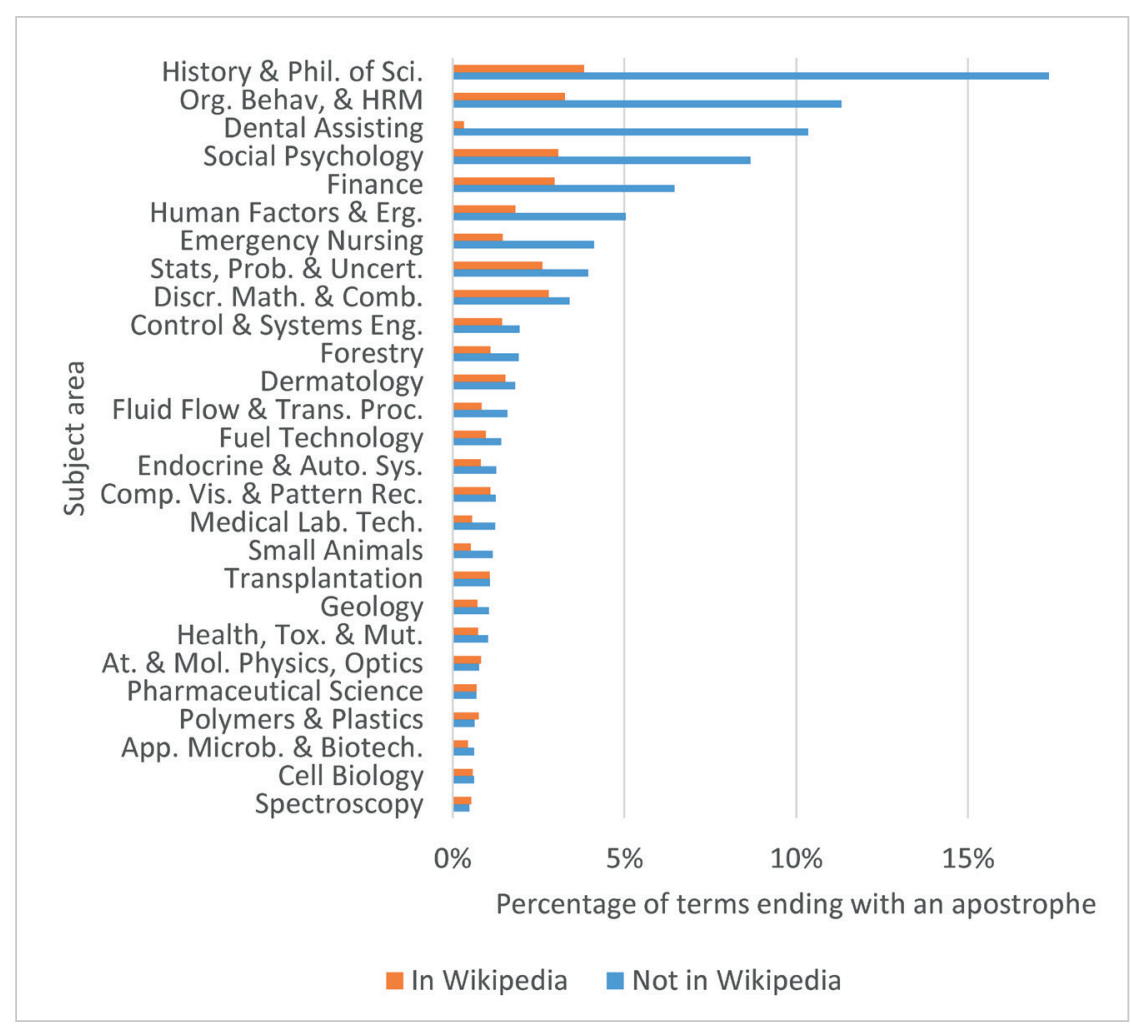

Figure 3. The percentage of terms in the titles of Scopus articles that end in an apostrophe or 's, normally indicating a possessive, broken down by whether they are also in Wikipedia.

although this is not true for the two topics that focus on anatomical parts of people: Dermatology and Transplantation. Finance fits this trend through its focus on actors in the financial sector (e.g., analysts', firms', auditors', management's, SEC's). As in the research process discussion below, for some categories possessives could be the names of academics, which effectively form a compound term with the entity that they are associated with. There were also mathematics and statistics terms that are grammaticaIly incorrect in standard English usage since they should not be used to pluralise acronyms (e.g., PDE's, as in "Analytic regularity and polynomial approximation of parametric and stochastic elliptic PDE's") and so not all the terms classed as possessives in this category are correctly classified (en.oxforddictionaries.com/punctuation/apostrophe). Overall, however, the possessive analysis supports the hypothesis of increased complexity for all titles outside of Wikipedia.

The simplest complexity comparison is to compare the length of words inside and outside of Wikipedia page titles in the full data set. In all cases, longer terms in Scopus titles tend not to be in Wikipedia page titles (Figure 4). Even allowing for the biasing effect 
of short common terms (e.g., it, the), the large numbers involved and the substantial differences support the complexity hypothesis. However, this may be largely a consequence of the general absence of hyphenated terms in Wikipedia page titles reported earlier, as hyphenation will necessarily result in increased average term length.

\subsection{Hypothesis 2: Research process descriptions}

Present participles had the strongest association with the research process and there is also an association for some possessives.

The present participle is relatively rare $(5 \%$ : Table 2$)$ but common in only one large subject. In History and Philosophy of Science (reconsidering, moderating, disentangling, historicizing, re-thinking, cointegrating, untangling), these present participles are used to describe how the author is approaching the topic investigated. These terms therefore indicate the research process, albeit in a very general way.

Some possessives (see above for the History and Philosophy of Science examples) also refer to aspects of the research process in the form of the name of the researcher. In the full data set this was evident in three areas. For Statistics, Probability and Uncertainty, possessives refer to researchers' methods (e.g., Kak's [Three-Stage Quantum Protocol], Zadeh's [fuzzy logic], Simes' [method]) or application areas (e.g., insurer's, firms'). For Discrete Mathematics and Combinatorics, possessives tend to refer to mathematicians' theories or unsolved problems (e.g., Heilbronn's [triangle problem], Gallai's [theorems], Thomassen's [conjecture], Hajos' [theorem]), analogously to the History and Philosophy of Science use of possessives for theories, as discussed above. Although these possessives can also indicate complexity, they may also serve to name something in the absence of a more logical invented term (abstract maths may not have many real-world referents to derive a name from) or to confer authority by naming an important researcher. The latter case relates indirectly to the research process.

Some suffix terms also denoted the research process in Polymers and Plastics, to some extent, by describing the process that had been applied to a molecule: compatibilizer, compatibilized, and plasticized.

\section{Journal article title terms may be absent from Wikipedia page titles due to stylis- tic differences although the underlying concepts are present in a different form}

Overall, there is strong evidence to support the research process description hypothesis as important in one area, History and Philosophy of Science, and weaker evidence for Statistics, Probability and Uncertainty, for Discrete Mathematics and Combinatorics, and for Polymers and Plastics.

\subsection{Hypothesis 3: Stylistic and structural differences}

Journal article title terms may be absent from Wikipedia page titles due to stylistic differences although the underlying concepts are present in a different form. The most obvious way in which this can occur is if one uses a singular form of a noun whereas the other uses the plural form. It can also occur if a term is not present in a Wikipedia page title but the stem term without any prefix or suffix is (see "Word variations" in Figure 1).

Plurals were a substantial minority overall (11\%: Table 2), except in Discrete Mathematics and Combinatorics (subgraphs, matchings, labelings, colourings, transversals, nonlinearities, subsequences, -graphs, edge-colorings, hyperovals, labellings, -factors, k-trees), where they were in most cases probably used as a generic term (e.g., "labellings for subgraphs" implies that a general method for labelling all subgraphs will be described). It may be primarily a stylistic issue that Wikipedia might use the singular form to denote generalisation in mathematics instead. A similar issue seems to occur in the vastly different field of Organizational Behavior and Human Resource Management (spillovers, adapt-abilities).

Terms with a prefix or suffix were also a substantial minority overall ( $17 \%$ combined, Table 2$)$. They were particularly common in Polymers and Plastics, including the three related prefix adjectives (diblock, triblock, and multiblock) and three related suffix adjectives, (compatibilizer, compatibilized, plasticized), as already mentioned.

The present tense is a large minority (10\%: Table 2$)$ that is common in two subjects and absent from most of the rest (14). In both Cell Biology (regulates, inhibits, enhances, modulates, interacts, stimulates, activates, suppresses, contributes, prevents, attenuates, impairs, encodes, facilitates, determines, confers, disrupts, potentiates) and Endocrine and Autonomic Systems (modulates, enhances, attenuates, regulates, inhibits, impairs, prevents, stimulates, suppresses, facilitates, contributes, modifies, potentiates, disrupts) present tense verbs are used to describe the actions of the entity investigated. Presumably if Wikipedia covers the topic of these articles then it would form part of an article on the entity rather than having a separate article on one of its actions. Thus, these terms probably reflect a focus on objects rather than actions in Wikipedia (nouns rather than verbs), a structural difference.

There are also some individual style differences in the use of hyphens and compound words. For example, although palmprint (Computer Vision and Pattern Recognition title frequency: 57) occurs in no Wikipedia page titles, there is a Wikipedia Palm print page. https://en.wikipedia.org/wiki/Palm_print

Similarly, for flowshop (Statistics, Probability and Uncertainty frequency: 46) with a Wikipedia Flow shop scheduling page. https://en.wikipedia.org/wiki/Flow_shop_scheduling

Acronyms and short forms of words may also be acceptable in journal article titles for an audience that would be familiar with them but not be present in Wikipedia pages. For example, Re-Os (Geology frequency 235) has an entire Wikipedia page called Rhenium-osmium dating. https://en.wikipedia.org/wiki/Rhenium-osmium_dating

Another example is surface electromyography (SEMG), which is described in a section in the Wikipedia electromyography (EMG) page.

https://en.wikipedia.org/wiki/Electromyography 


\subsection{Hypothesis 4: Culture bias}

Possessives could be markers of cultural bias if they referred to people that were of less interest in the English-speaking world. This only occurred in the History and Philosophy of Science and only for three people (Carnap's, Vygotsky's, Bourdieu's). None are native English speakers but all are widely known in the USA (Carnap moved there, and the others are internationally famous) and have large Wikipedia pages, so this is not strong evidence of cultural bias. In forestry, two terms refer to issues that are more important in warmer climates, silvopastoral (combining cattle and trees) and postfire (dealing with the aftereffects of devastating fires) but both are issues in the USA and discussed in this context in the literature.

In Geology, three terms were specific to China. Shahejie (in 115 journal article titles but no Wikipedia page titles) is a town and geological feature in China (e.g., "Diagenetic history and diagenetic stages prediction of Shahejie formation in the Qikou Sag"). For context, the word Shahejie occurs inside some English Wikipedia articles about Chinese railways and the only Wikipedia page with a title containing the term is Swedish, although Chinese Wikipedia contains the original term 沙河街, including a page dedicated to it.

https://sv.wikipedia.org/wiki/Shahejie_(h\%C3\%A4radshuvudort_i Kina,_Jiangxi_Sheng,_lat_29,61,_long_115,89

https://zh.wikipedia.org/wiki/沙河街镇

Tazhong (110) is an oil bearing area of China (e.g., "Oil and gas accumulations in the Ordovician carbonates in the Tazhong Uplift of Tarim Basin, west China"). Xujiahe (109) is a geological feature in China (e.g., "Analysis on provenance-supply system of Upper Triassic Xujiahe Formation, Sichuan basin"). This is the clearest evidence of cultural bias. In the small Dental Assisting category, the three Italian words in the top 25 are not relevant (frequency 1 each). In Emergency Nursing, Xuebijing (15) is a traditional Chinese medicine and all articles about it are written by Chinese authors. The term is not mentioned in a Wikipedia page from any language. The original term血必净 does not seem to be present in Chinese Wikipedia (this was double-checked by a native Chinese speaker) although it has a dedicated page in another Chinese online encyclopaedia.

https://wapbaike.baidu.com/item/血必净

No evidence of cultural bias was found in the other subjects.

There is evidence of cultural bias, albeit only against China and for Geology and Emergency Nursing

In conclusion, there is evidence of cultural bias, albeit only against China and for Geology and Emergency Nursing. The appearance of Chinese terms is probably due to a combination of the large size of the country and the indexing of some Chinese journals in Scopus, although the three terms found also occurred in international journals.

\subsection{Hypothesis 5: Editor gaps}

The three Chinese terms found above also serve as evidence of editor gaps. Presumably there were no experts on Shahejie, Tazhong or Xujiahe that were willing to write articles on them in the English Wikipedia. Given the existence of large national petrochemical companies, such as the China Petrochemical Corporation, it would make sense for the experts on these to be mainly resident Chinese.

The results confirm that there are gaps in the coverage of academic topics by Wikipedia

To search for specific editor gaps, the 675 terms were manually examined for terms, and particularly singular nouns, that might refer to topic areas absent from Wikipedia.

- Pharmaceutical Science: The brand name Eudragit (subject category frequency: 183) does not occur in any English Wikipedia page titles and only seems to occur in four English Wikipedia pages as a very minor mention in each case. https://en.wikipedia.org/wiki/Ethyl_acrylate

- Human Factors and Ergonomics: The concept of macroergonomics (14; macroergonomic: 7 ) is largely missing from Wikipedia, although it gets four passing mentions in the main Wikipedia article, two in method names as part of a list, and two in sentences starting with, "As applied to macroergonomics...". This is clear evidence of a research concept having no meaningful content in Wikipedia, although relatively low term frequencies are involved.

https://en.wikipedia.org/wiki/Human_factors_and_ ergonomics

- Small Animals: preantral (29) occurs only as two minor mentions in the page on folliculogenesis ("in contrast to so a called preantral follicle that still lacks an antrum") and is not mentioned in the page on antral follicles. This term is therefore implicitly defined but not discussed.

https://en.wikipedia.org/wiki/Folliculogenesis https://en.wikipedia.org/wiki/Antral_follicle

- Emergency Nursing: Fireground (33) is defined in Wikipedia but not extensively discussed. In academic articles, it seems to be a background term rather than a topic of discussion, however (e.g., "Establishing adequate fall protection on the fireground"). The Penehyclidine (11) [Hydrochloride] drug name is not in any Wikipedia page from any language version.

The above results confirm that there are gaps in the coverage of academic topics by Wikipedia.

It is possible that some areas have wider gaps in knowledge than others and a simple way to seek evidence of this is to assess whether any disciplines have a particularly high percentage of terms that are not in Wikipedia page titles. This is a very crude test, however, since term mismatches can be the result of many factors, as the above discussions show. The percentage of terms likely to be absent from Wi- 
kipedia is also likely to be statistically related to the size of a field and so points that are the highest above the trend line in Figure 5 are the most likely to have gaps. From this evidence, Discrete Mathematics and Combinatorics either has the most missing knowledge or other systematic factors, as described above. Pure mathematics might be the general area that is least accessible to a non-expert because of its hierarchical nature and extreme abstraction and so it would make sense if this subject was the least well represented in Wikipedia.

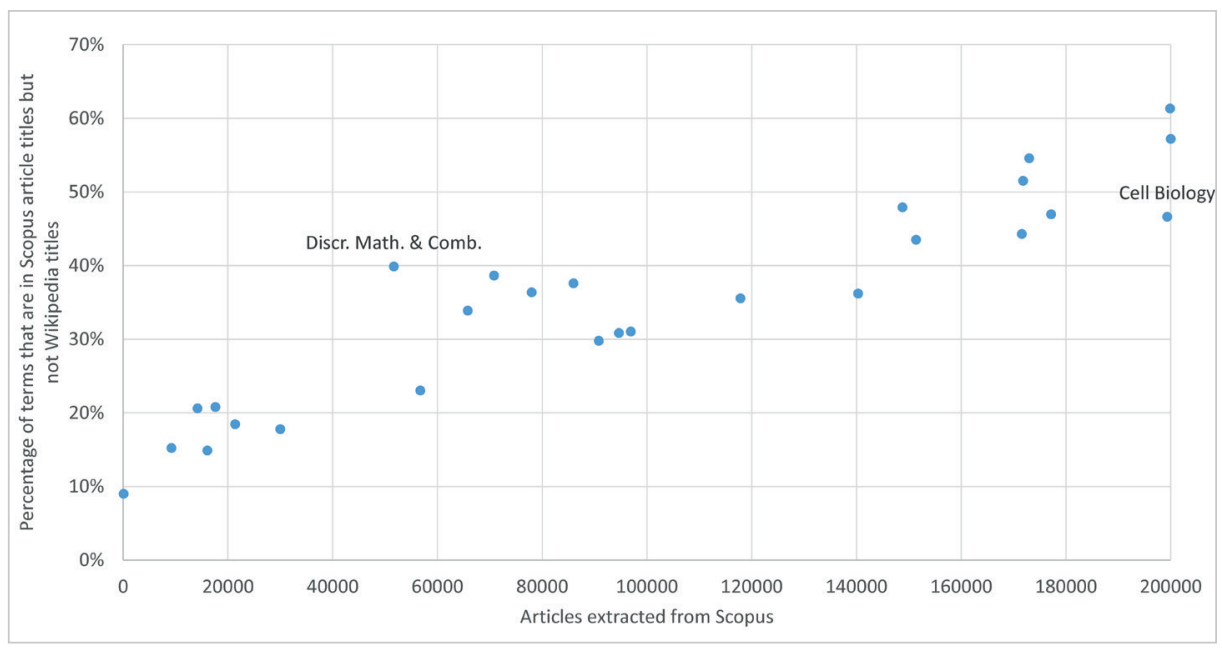

Figure 5. The percentage of terms that are in Scopus article titles but not in Wikipedia article titles against subject area size. The highest positive and negative outliers are named (identified from the residuals after linear regression).

\section{Limitations and discussion}

The results are limited in several respects in addition to those discussed in the methods section. Not all subject areas were analysed and the results may be different for those that were not chosen. The social sciences and humanities are not well represented in the fields covered and so important patterns in these areas have may been overlooked. The results are likely to be substantially different in a non-English Wikipedia and further research is needed to investigate this. Most importantly, the word frequency approach used here is indirect for the first four hypotheses and does not provide definitive evidence for them. Any failure to find evidence in support of a hypothesis is not evidence that the hypothesis is false for any subject. Moreover, since only the top 25 terms were examined for the primary analysis, the importance of one category of word for a subject would push out words from other categories, hiding the evidence that they provide. For the manual analysis, the choice of 25 terms rather than any other number is arbitrary and may have affected the results, as may the decision not to use word stemming.

\section{Article titles tend to be more complex} than Wikipedia page titles across all subjects analysed

The results tend to confirm the hypotheses, at least partiaIly, in all cases. In terms of the words contained in them, article titles tend to be more complex than Wikipedia page titles across all subjects analysed ( $\mathrm{H} 1)$, with longer terms and more hyphenated terms and, at least in the top 25, more compound words. This is consistent with Wikipedia carrying out the role of synthesising knowledge and simplifying and summarising it for a lay audience.

The strongest evidence of research process descriptions being absent from Wikipedia pages was in History and Philosophy of Science $(\mathrm{H} 2)$, where the missing terms described general analytical approaches, but there was also some evidence for three other areas. Presumably, the most common academic methods have their own Wikipedia pages and these are exceptions.

There was clear evidence of systematic stylistic or structural differences in four subject areas (H3), but not all. These included the use of plurals to signify abstraction in Discrete Mathematics and Combinatorics and Organizational Behavior and Human Resource Management, and the present tense to describe actions in both Cell Biology and Endocrine and Autonomic Systems that would be described within Wikipedia pages but would not have their own pages. There seem likely to be additional stylistic differences in all areas despite the failure to find more evidence.

\section{There was clear evidence of systematic stylistic or structural differences in four subject areas, but not all}

Although cultural biases may be present in other areas, they were only obvious in two, Geology and Emergency Medicine, and in both cases topics of interest mainly within China were absent from Wikipedia $(\mathrm{H} 4)$. There may well have been subtler cultural biases that were not identified, however, such as interest in chemicals because of their applications in local industries within a specific country. In this context and due to the difficulty in checking the background to the use of all terms, only areas that mention academics' names, geographic concepts, or localised professional practices would have a reasonable chance of producing obvious evidence of cultural biases.

Non-trivial coverage gaps were found in three subjects (H5) in terms of concepts that were not well covered in Wikipedia despite appearing frequently in academic journal titles (ignoring the minor Emergency Medicine example). These are in addition to the cultural bias gaps that are described above for two further areas, and the research description gaps found for a sixth. There were probably gaps in all areas but these results provide evidence that gaps do occur in Wikipedia. 


\section{Conclusions}

Wikipedia appears to be performing the role of a specialist encyclopaedia for all areas of academia, although it is imperfect by having gaps in some areas. Any other specialist encyclopaedia would presumably also have gaps, perhaps for the same reason (the lack of a willing specialist contributor). Thus, the main finding is that Wikipedia has wide coverage of academia, but with some omissions. Whilst extensive coverage of research must help it to reach a wider audience, it may cause users to rely on Wikipedia and not expect any important topics to be missing.

Wikipedia has wide coverage of academia, but with some omissions

Gaps in Wikipedia should not be a problem for field specialist academics who can find and read the original research if a simpler explanation is not available in Wikipedia. Other information seekers may turn to lower quality alternatives (Fallis, 2008), especially if they cannot understand or afford relevant scholarly publications or do not have the time to read them. There may also be negative consequences if decisions are made upon the apparently reasonable assumption that the absent information does not exist. For example, macroergonomic research might be ignored by managers that rely upon Wikipedia but have not heard of macroergonomics.

Given these potential negative consequences of topics missing from Wikipedia, academics should ensure that their specialism is adequately covered in Wikipedia. This especiaIly applies to topics that are useful for non-specialists who have not heard of them or could not find out about them from alternative sources.

English Wikipedia seems to include an element of cultural bias (not a pejorative term here), although this seems to be relatively minor. Perhaps academics that are not native English speakers but work in disciplines that publish primarily in English contribute to English Wikipedia and help to reduce its cultural bias. Thus, scholars should be careful to check areas that may be of less interest to academics in English speaking countries if the topics may be useful for non-academics that use English Wikipedia.

\section{Academics should ensure that their specialism is adequately covered in Wikipedia}

Research process descriptions seem to be well covered in Wikipedia, except for the generic terms of the History and Philosophy of Science (reconsidering, moderating, disentangling, historicizing, re-thinking, cointegrating, untangling). These seem to be describing analytical approaches that are too general to merit explicit description, however.

The stylistic and structural differences found in some subject areas confirm that academic research in Wikipedia is framed differently from in journal articles, with strong linguistic differences in some subject areas. When editing content, care should be taken to conform with the differing style within Wikipedia in comparison to academic English. Conversely, the evidence of stylistic differences presented here may also be useful to highlight to junior researchers that they need to adjust their language to cope with the differing formats required by specific subject areas. This may also be important for people conducting multidisciplinary research who may not notice that styles differ between fields.

Finally, librarians training information seekers should make them aware that gaps exist within Wikipedia, especially related to non-English speaking countries' culture and geography, but also, to a lesser extent, on any academic topic.

When editing content, care should be taken to conform with the differing style within Wikipedia in comparison to academic English

\section{References}

Azer, Samy A. (2015). "Is Wikipedia a reliable learning resource for medical students? Evaluating respiratory topics". Advances in physiology education, v. 39, n. 1, pp. 5-14. https://doi.org/10.1152/advan.00110.2014

Ball, Rafael (2009). "Scholarly communication in transition: The use of question marks in the titles of scientific articles in medicine, life sciences and physics 1966-2005". Scientometrics, v. 79, n. 3, pp. 667-679.

https://doi.org/10.1007/s11192-007-1984-5

Béjoint, Henri (2000). Modern lexicography: An introduction. Oxford, UK: Oxford University Press. ISBN: 0198299516

Burke, Ernest A. (2008). "Tidying up mineral names: an IMA-Cnmnc scheme for suffixes, hyphens and diacritical marks". Mineralogical record, v. 39, n. 2, pp. 131-135. http://ima-cnmnc.nrm.se/tidyingupnames.pdf

Callahan, Ewa; Herring, Susan C. (2011). "Cultural bias in Wikipedia content on famous persons". Journal of the American Society for Information Science and Technology, v. 62, n. 10, pp. 1899-1915.

https://doi.org/10.1002/asi.21577

Cho, Hichang; Chen, Meihui; Chung, Siyoung (2010). "Testing an integrative theoretical model of knowledge-sharing behavior in the context of Wikipedia". Journal of the Association for Information Science and Technology, v. 61, n. 6, pp. 1198-1212.

https://goo.gl/sDxL2c

https://doi.org/10.1002/asi.21316

Cohen, Jacob (1960). "A coefficient of agreement for nominal scales". Educational and psychological measurement, v. 20, n. 1, pp. 37-46.

https://doi.org/10.1177/001316446002000104

Cook, James; Plourde, Dawn (2016). "Do scholars follow Betteridge's Law? The use of questions in journal article titles". 
Scientometrics, v. 108, n. 3, pp. 1119-1128.

https://goo.gl/VNu99t

https://doi.org/10.1007/s11192-016-2030-2

De-Laat, Paul (2012). "Open source production of encyclopedias: Editorial policies at the intersection of organizational and epistemological trust". Social epistemology, v. 26, n. 1, pp. 71-103.

https://philpapers.org/archive/DELOSP.pdf

https://doi.org/10.1080/02691728.2011.605478

Dick, Bob (2015). "Reflections on the SAGE Encyclopedia of Action Research and what it says about action research and its methodologies". Action research, v. 13, n. 4, pp. 431-444. https://goo.gl/ziRxPh

https://doi.org/10.1177/1476750315573593

Fallis, Don (2008). "Toward an epistemology of Wikipedia". Journal of the Association for Information Science and Technology, v. 59, n. 10, pp. 1662-1674.

https://papers.ssrn.com/so/3/papers.cfm?abstract $i d=1263781$

https://doi.org/10.1002/asi.20870

Flanagin, Andrew J.; Metzger, Miriam J. (2011). "From Encyclopaedia Britannica to Wikipedia: Generational differences in the perceived credibility of online encyclopedia information". Information, communication \& society, v. 14, n. 3, pp. 355-374.

https://goo.gl/irg1DH

https://doi.org/10.1080/1369118X.2010.542823

Frey-Klett, Pascale; Burlinson, Peter; Deveau, Aurélie; Barret, Matthieu; Tarkka, M.; Sarniguet, Alain (2011). "Bacterial-fungal interactions: hyphens between agricultural, clinical, environmental, and food microbiologists". Microbiology and molecular biology reviews, v. 75, n. 4, pp. 583-609.

https://doi.org/10.1128/MMBR.00020-11

Giles, Jim (2005). "Internet encyclopaedias go head to head". Nature, v. 438, n. 7070, pp. 900-901.

https://doi.org/10.1038/438900a

Gill, Frank B.; Wright III, Minturn T.; Conyne, Sally B.; Kirk, Robert (2009). "On hyphens and phylogeny". The Wilson journal of ornithology, v. 121, n. 3, pp. 652-655.

https://goo.gl/z6n9hi

Gorichanaz, Tim (2016). "How the document got its authority". Journal of documentation, v. 72, n. 2, pp. 299-305.

https://doi.org/10.1108/JD-09-2015-0117

Gralla, Preston (2009). "What was Encarta? Look it up on Wikipedia". PCWorld, 31 Mar.

http://www.pcworld.com/article/162320/what_was_encarta_ look_it_up_on_wikipedia.html

Halavais, Alexander; Lackaff, Derek (2008). "An analysis of topical coverage of Wikipedia". Journal of computer-mediated communication, v. 13, n. 2, pp. 429-440.

https://doi.org/10.1111/j.1083-6101.2008.00403.x

Hartley, James (2005). "To attract or to inform: what are titles for?". Journal of technical writing and communication, v. 35, n. 2, pp. 203-213.

https://doi.org/10.2190/NV6E-FN3N-7NGN-TWQT
Hartley, James (2007). "There's more to the title than meets the eye: Exploring the possibilities". Journal of technical writing and communication, v. 37, n. 1, pp. 95-101.

https://goo.gl/wWsM4r

https://doi.org/10.2190/BJ16-8385-7Q73-1162

Hasty, Robert T.; Garbalosa, Ryan C.; Barbato, Vicenzo A.; Valdes Jr, Pedro J.; Powers, David W.; Hernandez, Emmanuel; John, Jones S.; Suciu, Gabriel; Qureshi, Farheen; Poparadu, Matei; San-José, Sergio; Drexler, Nathaniel; Patankar, Rohan; Paz, José R.; King, Christopher; Gerber, Hilary N.; Valladares, Michael G.; Somji, Alyaz A. (2014). "Wikipedia vs peer-reviewed medical literature for information about the 10 most costly medical conditions". Journal of the American Osteopathic Association, v. 114, n. 5, pp. 368-373. https://doi.org/10.7556/jaoa.2014.035

Henderson, Michael; Selwyn, Neil; Finger, Glenn; Aston, Rachel (2015). "Students' everyday engagement with digital technology in university: exploring patterns of use and 'usefulness'". Journal of higher education policy and management, v. 37, n. 3, pp. 308-319.

https://goo.gl/cWJWu4

https://doi.org/10.1080/1360080X.2015.1034424

Hughes, Benjamin; Joshi, Indra; Lemonde, Hugh; Wareham, Jonathan (2009). “Junior physician's use of Web 2.0 for information seeking and medical education: a qualitative study". International journal of medical informatics, v. 78, n. 10, pp. 645-655. https://doi.org/10.1016/j.ijmedinf.2009.04.008

Jamali, Hamid R.; Nikzad, Mahsa (2011). "Article title type and its relation with the number of downloads and citations". Scientometrics, v. 88, n. 2, pp. 653-661. https://doi.org/10.1007/s11192-011-0412-z

Kousha, Kayvan; Thelwall, Mike (2017). "Are Wikipedia citations important evidence of the impact of scholarly articles and books?". Journal of the Association for Information Science and Technology, v. 68, n. 3, pp. 762-779.

https://goo.gl/YHnCgF

https://doi.org/10.1002/asi.23694

Kubiszewski, Ida; Noordewier, Thomas; Costanza, Robert (2011). "Perceived credibility of Internet encyclopedias". Computers \& education, v. 56, n. 3, pp. 659-667.

https://www.uvm.edu/giee/pubpdfs/Kubiszewski_2011_ Computers_and_Education.pdf https://doi.org/10.1016/j.compedu.2010.10.008

Lærke, Mogens (2014). "Leibniz, the encyclopedia, and the natural order of thinking". Journal of the history of ideas, v. 75, n. 2, pp. 237-259.

Lai, Cheng-Yu; Yang, Heng-Li (2014). "The reasons why people continue editing Wikipedia content-task value confirmation perspective". Behaviour \& information technology, v. 33, n. 12, pp. 1371-1382.

https://doi.org/10.1080/0144929X.2014.929744

Landis, J. Richard; Koch, Gary G. (1977). "The measurement of observer agreement for categorical data". Biometrics, v. 33 , n. 1 , pp. 159-174.

https://doi.org/10.2307/2529310 
Lange, Jessica (2016). "Scholarly management publication and open access funding mandates: a review of publisher policies". Ticker: The academic business librarianship review, v. 1, n. 3, pp. 15-27.

https://goo.gl/6aaGgW

Lim, Sook (2009). "How and why do college students use Wikipedia?". Journal of the Association for Information Science and Technology, v. 60, n. 11, pp. 2189-2202.

https://goo.gl/muAGJf

https://doi.org/10.1002/asi.21142

Loveland, Jeff (2013). "Encyclopaedias and genre, 1670-1750". Journal for eighteenth-century studies, v. 36, n. 2, pp. 159-175.

https://goo.gl/jMhJrx

https://doi.org/10.1111/j.1754-0208.2012.00493.x

Luyt, Brendan (2015). "Debating reliable sources: writing the history of the Vietnam War on Wikipedia". Journal of documentation, v. 71, n. 3, pp. 440-455.

https://doi.org/10.1108/JD-11-2013-0147

Masukume, Gwinyai; Kipersztok, Lisa; Das, Diptanshu; Shafee, Thomas; Laurent, Michaël R.; Heilman, James M. (2016). "Medical journals and Wikipedia: A global health matter". Lancet global health, v. 4, e791.

http://dx.doi.org/10.1016/S2214-109X(16)30254-6

Mathew, Manu E.; Joseph, Anna; Heilman, James M.; Tharyan, Prathap (2013). "Cochrane and Wikipedia: the collaborative potential for a quantum leap in the dissemination and uptake of trusted evidence". The Cochrane database of systematic reviews, v. 10, ED000069.

https://doi.org/10.1002/14651858.ED000069

Méndez, David I.; Alcaraz, María-Ángeles; Salager-Meyer, Françoise (2014). "Titles in English-medium astrophysics research articles". Scientometrics, v. 98, n. 3, pp. 2331-2351.

http://rua.ua.es/dspace/handle/10045/46404

https://doi.org/10.1007/s11192-013-1174-6

Mesgari, Mostafa; Okoli, Chitu; Mehdi, Mohamad; Nielsen, Finn-Årup; Lanamäki, Arto (2015). "'The sum of all human knowledge': A systematic review of scholarly research on the content of Wikipedia". Journal of the Association for Information Science and Technology, v. 66, n. 2, pp. 219-245. https://spectrum.library.concordia.ca/978652/ https://doi.org/10.1002/asi.23172

Messner, Marcus; DiStaso, Marcia W. (2013). "Wikipedia versus Encyclopedia Britannica: A longitudinal analysis to identify the impact of social media on the standards of knowledge". Mass communication and society, v. 16, n. 4, pp. 465-486.

https://goo.gl/Wh8G4q

https://doi.org/10.1080/15205436.2012.732649

Nielsen, Finn-Årup (2007). Scientific citations in Wikipedia. First Monday, v. 12, n. 8.

http://firstmonday.org/article/view/1997/1872

https://arxiv.org/pdf/0705.2106.pdf

Nov, Oded (2007). "What motivates Wikipedians?". Communications of the ACM, v. 50, n. 11, pp. 60-64.

https://goo.gl/Fj6fhE
OED (2016). Oxford English dictionary (online).

http://www.oed.com

Orduña-Malea, Enrique; Thelwall, Mike; Kousha, Kayvan (2017). "Web citations in patents: Evidence of technological impact?". Journal of the Association for Information Science and Technology, v. 68, n. 8, pp. 1967-1974.

https://doi.org/10.1002/asi.23821

Paiva, Carlos-Eduardo; Lima, João-Paulo-Da-Silveira-Nogueira; Paiva, Bianca-Sakamoto-Ribeiro (2012). "Articles with short titles describing the results are cited more often". Clinics, v. 67, n. 5, pp. 509-513.

https://doi.org/10.6061/clinics/2012(05)17

Pinfield, Stephen; Salter, Jennifer; Bath, Peter A. (2016). "The 'total cost of publication' in a hybrid open-access environment: Institutional approaches to funding journal article-processing charges in combination with subscriptions". Journal of the Association for Information Science and Technology, v. 67, n. 7, pp. 1751-1766.

https://doi.org/10.1002/asi.23446

Remy, Charlie (2015). “Oxford's research encyclopedias: A new model for reference content?". Journal of electronic resources librarianship, v. 27, n. 3, pp. 204-210. https://doi.org/10.1080/1941126X.2015.1059663

Roessing, Thomas; Einwiller, Sabine (2016). "Portrayals of large corporations in the English and German version of Wikipedia -Exploring similarities and differences". Corporate reputation review, v. 19, n. 2, pp. 108-126.

https://goo.gl/nMmsHc

https://doi.org/10.1057/crr.2016.3

Rostami, Fatemeh; Mohammadpoorasl, Asghar; Hajizadeh, Mohammad (2014). "The effect of characteristics of title on citation rates of articles". Scientometrics, v. 98, n. 3, pp. 2007-2010.

https://doi.org/10.1007/s11192-013-1118-1

Royal, Cindy; Kapila, Deepina (2009). "What's on Wikipedia, and what's not...? Assessing completeness of information". Social science computer review, v. 27, n. 1, pp. 138-148. https://doi.org/10.1177/0894439308321890

Rush, Elizabeth K.; Tracy, Sara J. (2010). "Wikipedia as public scholarship: Communicating our impact online". Journal of applied communication research, v. 38, n. 3, pp. 309-315. https://goo.gl/WoX2Lx

https://doi.org/10.1080/00909882.2010.490846

Samoilenko, Anna; Yasseri, Taha (2014). "The distorted mirror of Wikipedia: a quantitative analysis of Wikipedia coverage of academics". EPJ data science, v. 3, n. 1, pp. 1. https://doi.org/10.1140/epjds20

Sano, Hikomaro; Fujiwara, Yuzuru (1993). "Syntactic and semantic structure analysis of article titles in analytical chemistry". Journal of information science, v. 19, n. 2, pp. 119124.

https://doi.org/10.1177/016555159301900203

Serrano-López, Antonio-Eleazar; Ingwersen, Peter; Sanz-Casado, Elías (2017). "Wind power research in Wikipedia: Does Wikipedia demonstrate direct influence of research publications and can it be used as adequate source 
in research evaluation?". Scientometrics, v. 112, n. 3, pp. pp. 1471-1488.

https://doi.org/10.1007/s11192-017-2447-2

Signore, Angelo; Serio, Francesco; Santamaria, Pietro (2014). "Wikipedia as a tool for disseminating knowledge of (agro) biodiversity". HortTechnology, v. 24, n. 1, pp. pp. 118-126.

http://horttech.ashspublications.org/content/24/1/118. abstract

Soler, Viviana (2011). "Comparative and contrastive observations on scientific titles written in English and Spanish". English for specific purposes, v. 30, n. 2, pp. 124-137.

https://doi.org/10.1016/j.esp.2010.09.002

Stankus, Tony; Spiegel, Sarah E. (2010). "Wikipedia, Scholarpedia, and references to books in the brain and behavioral sciences: A comparison of cited sources and recommended readings in matching free online encyclopedia entries". Science \& technology libraries, v. 29, n. 1-2, pp. 144-164. https://doi.org/10.1080/01942620903579435

Swales, John (1990). Genre analysis: English in academic and research settings. Cambridge, UK: Cambridge University Press. ISBN: 9780521338134

Szajewski, Michael (2013). "Using Wikipedia to enhance the visibility of digitized archival assets". DLib magazine, v. 19, n. 3/4, pp. 1-8.

https://doi.org/10.1045/march2013-szajewski

Teplitskiy, Misha; Lu, Grace; Duede, Eamon (2017). “Amplifying the impact of open access: Wikipedia and the diffusion of science". Journal of the Association for Information Science and Technology, v. 68, n. 9, pp. 2116-2127.

https://arxiv.org/abs/1506.07608

https://doi.org/10.1002/asi.23687

Thelwall, Mike (2016). "Does astronomy research become too dated for the public? Wikipedia citations to astronomy and astrophysics journal articles 1996-2014". El profesional de la información, v. 25, n. 6, pp. 893-900.

https://doi.org/10.3145/epi.2016.nov.06

Thomas, Garry R.; Eng, Lawson; De-Wolff, Jacob F.; Grover, Samir C. (2013). "An evaluation of Wikipedia as a resource for patient education in nephrology". Seminars in dialysis, v. 26, n. 2, pp. 159-163. https://doi.org/10.1111/sdi.12059

Ubriani, Ravi; Smith, N.; Katz, Kenneth A. (2007). "Reporting of study design in titles and abstracts of articles published in clinically oriented dermatology journals". British journal of dermatology, v. 156, n. 3, pp. 557-559.

https://doi.org/10.1111/j.1365-2133.2006.07705.x

Wang, Yan; Bai, Yongquan (2007). "A corpus-based syntactic study of medical research article titles". System, v. 35, n. 3, pp. 388-399.

https://goo.gl/xZ8U7G

https://doi.org/10.1016/j.system.2007.01.005

White, Arden; Hernandez, Nelda-Rae (1991). "Increasing field complexity revealed through article title analyses". Journal of the American Society for Information Science, v. 42 , n. 10, pp. 731-734.

https://doi.org/10.1002/(SICI)1097-4571(199112)42:10<731::AIDASI6>3.0.CO;2-W

Wilson, Adam; Likens, Gene E. (2015). "Content volatility of scientific topics in Wikipedia: a cautionary tale". PloS one, v. 10, n. 8, e0134454.

https://doi.org/10.1371/journal.pone.0134454

Wodak, Shoshana J.; Mietchen, Daniel; Collings, Andrew M.; Russell, Robert B.; Bourne, Philip E. (2012). "Topic pages: PLoS computational biology meets Wikipedia". PLOS computational biology, v. 8, n. 3, e1002446.

https://doi.org/10.1371/journal.pcbi.1002446

Xu, Bo; Li, Dahui (2015). "An empirical study of the motivations for content contribution and community participation in Wikipedia". Information \& management, v. 52, n. 3, pp. 275-286.

https://goo.gl/NkMFX3

https://doi.org/10.1016/j.im.2014.12.003

Yam, Shing-Chung J. (2016). "Negotiating boundaries of knowledge: Discourse analysis of Wikipedia's Articles for Deletion (AfD) discussion". Communication and critical/cultural studies, v. 13, n. 3, pp. 305-323.

https://doi.org/10.1080/14791420.2015.1137334

Yang, Heng-Li; Lai, Cheng-Yu (2010). "Motivations of Wikipedia content contributors". Computers in human behavior, v. 26 , n. 6, pp. 1377-1383.

https://doi.org/10.1016/j.chb.2010.04.011

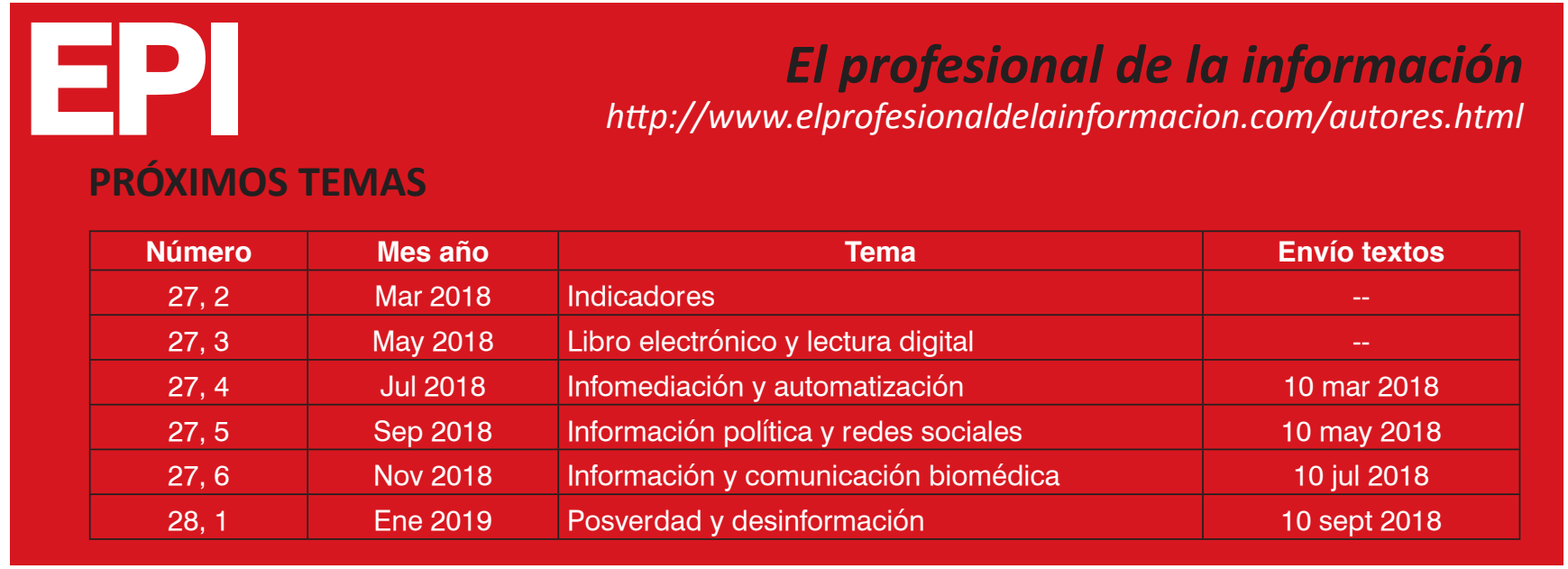

